\title{
PERSPECTIVE
}

\section{Artificial Intelligence: Lessons Learned from Radiology}

\author{
Elizabeth A. Krupinski, PhD*
}

\begin{abstract}
Artificial intelligence (Al) will continue to increase its significant impact on the everyday acquisition, interpretation, and application of data in our healthcare system. It is difficult to predict whether and to what extent Al will change the health status of patients, but it certainly will change the way decisions are made, how healthcare is delivered, and the ways providers, patients, and healthcare enterprises interact with and use data from an increasing array of sources. Radiology has often been at the forefront of technological change in healthcare and the Al revolution is no different. The lessons radiology has learned to date, its successes and challenges, can help provide guidance for other specialties, especially since radiology interacts with and cross-cuts nearly every other specialty and touches nearly every patient at least once in his or her lifetime.
\end{abstract}

\section{Introduction}

Artificial intelligence (AI) and its related application techniques (e.g., deep learning, machine learning, and convolutional neural networks) are increasingly becoming a key component of healthcare technologies, clinical decision making, and everyday workflow. Although it is impossible to say what was the first AI application in medicine, it is likely safe to say that radiology has been at the forefront of AI research and clinical applications. This stems from three key reasons: (1) the fact that radiology is inherently a specialty that has always relied on technology and technological advances, (2) the fact that radiology as a clinical specialty cross-cuts nearly all of healthcare and other clinical specialties and, therefore, requires a high degree of integration and ways to convey results in meaningful and actionable ways, and (3) the fact that its information systems (e.g., Picture Archiving and Communications Systems [PACS], Radiology Information Systems) have been the cornerstones of integrating data in the electronic health record (EHR). Thus, radiology has a large body of research and practical clinical experience in AI that other specialties could benefit from.

Why is AI becoming so important in healthcare? Much of it has to do with the prevalence of errors in clinical decision making as well as at nearly every possible point of contact patients have with the healthcare system ${ }^{1,2}$ and growing concerns about cost and ways that new technologies (e.g., robots) can help reduce them. ${ }^{3}$ Error has been studied in depth in radiology for $>60$ years and technology has advanced considerably, yet fundamental error rates have not changed significantly here or in most other clinical specialties. ${ }^{4-14}$ Why? Humans make errors. The information available to render diagnostic and treatment decisions is often incomplete, incredibly variable, unavailable, and inaccurate. Health providers vary in type of training, level of experience and expertise, exposure to different types of patients and conditions, knowledge, and individual skills and art of practicing medicine. This has all been exacerbated in recent years by the incessant increase in the amount and variability of information available to providers from a wide variety of technologies, devices, tools, and research articles, as well as the growing complexity of healthcare systems, aging populations, patients with complex and chronic conditions, and long workdays that lead to fatigue and burnout. ${ }^{15-20}$ Quite simply_our healthcare enterprises need help and AI may just be a powerful enough tool to provide that help.

In radiology, potential applications run the gamut from automatic detection of diseases and other findings, automatically measuring changes in tumor volumes as a function of treatment, creating imaging protocols, reducing dose, and improving image quality, decreasing scan times for advanced modalities such as computed tomography (CT) and magnetic resonance imaging (MRI), optimizing technologists' time and scanner utilization, use of triaging algorithms (e.g., triage out likely normal 
mammograms to not read or read later to focus on abnormal and indeterminate cases; moving CT head examinations with likely strokes to the top of the list; or moving images with aortic dissections and ruptures to the top of the list), use of natural language processing (NLP) to "read" and analyze reports for mistakes and inconsistencies or "rewrite" them for consistency and improved communicability for other providers and patients, use of NLP for automatic coding and assignment of billing codes based on report content, and developing better training, education, and assessment tools.

\section{Al and Computer-Aided Detection/Diagnosis}

$\mathrm{AI}$ as a tool to analyze medical images has its roots in radiology's computer-aided detection (CAD) and computeraided diagnosis (CADx) schemes that started to develop quite quickly with the advent of digital radiography and PACS in the latter part of the 20th and early $21 \mathrm{st}$ centuries. $^{21-24}$ The promise and the hype we are experiencing with AI was there with CAD. Everyone thought it would significantly reduce error rates, reduce image interpretation times, catch cancers earlier, and yes-even take away radiologists' jobs. Nearly every major academic radiology department had someone doing CAD research, many small companies were formed (and many failed), big companies invested in the research and development, the Food and Drug Administration (FDA) invested in teams that could develop ways to evaluate CAD tools for eventual approval, and a number of schemes made it into routine clinical practice. Perhaps the most well known being mass and microcalcification detection in mammographic imaging (starting in 1998), ${ }^{21,23}$ polyp detection in virtual colonoscopy studies, ${ }^{25}$ and lung nodule detection in chest images. ${ }^{26}$

\section{Radiologists' Perceptions About Al}

AI is inevitable. The real question is, however, what exactly will it do and how much of what a healthcare provider does will be done by AI-will it really take away jobs or even eliminate certain specialties like radiology ? $^{27-29}$ Views are mixed. An interesting study by Goldberg et al. ${ }^{30}$ examined one of the most popular social media platforms, Twitter, to gauge the public's perceptions about AI. They searched for tweets containing "artificial intelligence" and "radiology" over a 1-year period (November 2016-October 2017), and categorized those posting tweets, content, and linked websites. They found 605 tweets from 407 unique users with most from industry $(22.6 \%)$ and only $9.3 \%$ from radiologists. A favorable stance regarding the impact of AI on radiology was reflected in $25 \%, 75 \%$ were neutral, and none unfavorable. Of the websites linked to, the majority $(88 \%)$ leaned toward AI being positive for radiology and none were negative. The most common website link themes were AI-related efficiency and/or workflow improvements $(52 \%)$, challenges $(29 \%)$, legal and regulatory issues (18\%), and ethical issues (5\%). Regarding AI replacing radiologists, $4 \%$ websites leaned toward it, $37 \%$ against $\mathrm{AI}, 6 \%$ presented both sides, and $53 \%$ did not note.

Medical trainees may be more open to the prospect of working with AI in their future careers than those practicing today. Pintos dos Santos et al. ${ }^{31}$ surveyed medical students $(n=263)$ at three major medical schools and $52 \%$ were aware of AI discussions in radiology, although $68 \%$ were unaware of the technologies involved. The majority $(83 \%)$ thought AI could likely detect pathologies on imaging, but $56 \%$ thought it was unlikely AI could provide a definitive diagnosis. Overall, 77\% thought AI will revolutionize radiology and $86 \%$ thought it will improve it. On positive note, $83 \%$ disagree that AI will replace radiologists and $71 \%$ agreed it should be incorporated into medical training.

There are also issues in gaining acceptance and adoption of AI across the healthcare enterprise, ranging from the C-suite and department chairs to state medical boards to the Centers for Medicare and Medicaid Services (CMS) and the FDA. It took years to get CAD tools approved by the FDA and CADx tools are still lagging in approval. AI tools are simply more advanced versions of CAD and CADx for the most part and are accelerating in development much faster than CAD and CADx ever did. There are some doubts about whether the FDA can keep up and whether these tools can be successfully approved and integrated into clinical practice before they become outdated. On a positive note, in recent months, the FDA has been addressing this issue and changing some of its procedures in line with a recent framework document. ${ }^{32}$ There are also products being developed that continuously learn as they get exposed to more data. This brings up the question, however, as to whether a system that continuously learns and updates its capabilities is the same as the one that was approved. An added problem is how and whether users would need to be informed about what the new "status" of the tool is and whether the thresholds for various measurements, parameters, decisions, and so on have changed, thus impacting how the tools are incorporated into the decision-making process.

\section{Some Key Challenges}

Although there are more and more AI techniques being developed that require fewer images and/or other data sources to be "properly" trained, validated, and tested, 
to include the immense range of variation of abnormal and normal in medical images and other data, massive amounts of data are required. This article will not deal with the technical challenges involved in creating AI schemes for radiology and medicine in general as there is a very large body of literature on this topic (e.g., overtraining, over/underfitting, "black box," bias, and imbalanced learning). ${ }^{33-35}$

Vayena et al. ${ }^{36}$ nicely summarize some key ethical areas that impact the acquisition and curation of these vast data sets: (1) data sourcing must adhere to data protection and privacy requirements, (2) scheme development should be committed to fairness (no biases), and (3) development should satisfy transparency requirements (the "black box" problem). To some extent (2) and (3) are more technical in nature and perhaps easier to address. The first topic, however, is quite a difficult challenge and in radiology it poses some interesting aspects that are rather unique.

For example, CT and MRI are volumetric modalities that allow for three-dimensional (3D) rendering of the acquired data, the addition of pseudocoloring, the ability to visualize soft tissues and skin lines over underlying bones and organs, and then visualization of an amazing reconstruction on a traditional computer display or in a virtual or augmented reality environment. The clinical uses are endless, exciting, and clearly have already started to impact areas such as surgery and radiation oncology. So, what is the downside? Imagine all of this being done with a CT or MRI of the head. Now imagine that very real face that emerges from data that even without any protected health information (PHI) is quite identifiable. In a fascinating study by Prior et al., ${ }^{37}$ they demonstrated just how real this problem could be. They used 3D reconstructions of MR images and asked subjects to match them to a series of photographs. Forty percent of the subjects were able to accurately match the MR images with the proper photograph of the person imaged!

It does not apply only to faces. Although many diseases and problems seem to be quite common and to many people look to be the same, unique presentations are quite common and readily traceable with only a few other bits of PHI back to a specific patient. Even something as innocent as a bracelet or necklaces captured on an X-ray image or less often thought of like serial numbers on implanted devices can readily identify a patient. If data need to be completely deidentified before being used by someone for training, validating, and testing an AI scheme, is it even feasible?

What level of deidentification should we require? Right now, most agree that at a minimum, all digital imaging and communications in medicine (DICOM) infor- mation must be removed from radiology and other images that use DICOM formats. The problem is that there is not just one vanilla DICOM (even though that was the original intent). Nearly every vendor has some proprietary data tags, the DICOM fields are located in slightly different places or contain slightly different information. The same holds for information in electronic patient records-even if two institutions use the same company for their medical record, they could have different versions, different modules, custom interfaces, and/or fields and so on. This makes automatic deidentification of data elements rather difficult and manual scrubbing is essentially impractical when talking about the vast amounts of data $\mathrm{AI}$ algorithms require.

Many of the challenges are actually on the implementation side. The most obvious challenge is creating an interface and work environment that truly integrates AI into the clinical workflow. Although this might sound easy, it really is not. Integration of CAD and other decision support tools into the radiology work environment has been a challenge since it first found its way into clinical use and we are still trying to find the best way(s) to present computer-based information to radiologists that will efficiently and efficaciously impact their decision-making processes. ${ }^{38}$ The Academy for Radiology and Biomedical Imaging Research has been working for the past few years on the concept of the "diagnostic cockpit of the future." 39 The concept is of a "future-state digital platform to aggregate, organize, and simplify medical imaging results and patient-centric clinical data ... (for) detecting disease early, making accurate diagnoses, driving imageguided interventions, and improving downstream clinical management of patients." One of the key challenges the effort faces is lack of standards and standardization in the wide variety of components of the patient record (including imaging) and history that $\mathrm{AI}$ algorithms need to operate on to create a seamless working environment that synergistically provides information to radiologists and other healthcare providers. The workshops held so far on the diagnostic cockpit have, be necessity, brought together representatives from academia, healthcare systems, industry, and the government (e.g., National Institute of Standards and Technology, National Institute of Biomedical Imaging and Bioengineering).

One topic brought up at a recent meeting of the Medical Image Perception Society (MIPS) that raises some interesting questions is the impact of AI on the actual cognitive processes underlying medical decisions. For example, in mammography screening, mammographers read hundreds of images per day with the overwhelming majority being normal (breast cancers are found in most screening centers about 1 in every 1000 cases). Thus, an 
ideally suited task for AI would be to scan all screening mammograms and triage out likely normal mammograms to not read or read later so mammographers could focus on abnormal and indeterminate cases. This would also help reduce the fatigue associated with reading so many cases and the likelihood of missing relatively rare findings. Sounds great - so what is the problem? The problem is that this triaging out of so many cases changes the density mix, prevalence rates, and so on. This will necessitate a change in detection and reporting thresholds that the mammographers use in their decision-making processes. We are going to need to educate users about these changes and what it does to their perceptual and cognitive systems.

There are also concerns that algorithms may adopt/ learn existing human biases in decision making based on factors such as age, gender, and ethnicity. For example, there are algorithms being developed to predict which hospital would be best to direct an ambulance with a given patient to (e.g., stroke, heart attack, and traffic accident) based on factors such as distance, scanner type, and scanner availability. Ideally the scheme should send the patient to the best hospital with the most readily available scanner and required personnel. However, with access to information such as patient's home address, age, ethnicity, and insurance type of coverage decisions could be biased to send only those with the means to afford the most appropriate or best care to the "right" facilities. Instead of basing decisions on patient needs and the best care, decisions could be driven by actions that would more likely generate a profit. The ethical issues are complex. For an excellent summary of ethical and regulatory issues in the United States and Europe, see Pesapane et al. $^{40}$ and added perspective from the United Kingdom by Wong et al. ${ }^{41}$

\section{Opportunities}

There are plentiful opportunities for integrating radiology AI applications into health system-wide applications. PACS were originally developed as a standalone application and only later were integrated into EHRs. This raises the intriguing possibility that as AI tools continue to develop and as those schemes that learn are exposed to more data become dominant, AI apps for radiology can also be used for other image-based specialties such as pathology, dermatology, and ophthalmology. There is also the possibility that since all of these other specialties are also developing AI tools, the schemes could learn from each other and "merge" into a more unified AI tool.

Also intriguing is the opportunity to expand AI beyond simple detection and classification into a more predictive cross-specialty decision-support system, which would be even more powerful with the type of merged system already noted. For example, suppose a radiology AI system identifies a malignant mass in a breast image and a pathology AI system determines exactly what type of cancer it is, an oncology AI system identifies the best treatment based on the patient's individual data compared with large patient data sets, and an administrative AI system schedules all necessary appointments using the hospital's scheduling system and the patient's schedule as acquired from the patient portal.

There are also potential implications for consumer health with home-based technologies and retail health. There are already ultrasound devices that are affordable for consumers and targeted directly for home use. For example, an Israeli company has developed a system that can be used by a woman to scan her breasts every month, send the image data to a cloud-based analysis system that compares the image data with other images in its database (as well as the woman's own prior images), and detects any potential signs of cancer. The app then notifies the user, who can then schedule an appointment to get appropriate follow-up. ${ }^{42}$

\section{Where to Learn About Al in Radiology}

Although image analysis and automated decision-making tools have been a part of radiology for $>30$ years, the AI buzz started to really take hold $<10$ years ago, but since then it has risen exponentially. There is probably no radiology meeting one could go to today that does not have multiple talks about AI, numerous vendors promoting AI-based products, and attendees looking to learn more through workshops, talks, and major keynote presentations. The world's largest radiology meeting in the Radiological Society of North America (RSNA) is held every year in Chicago, IL, the week after Thanksgiving (https://www.rsna.org/annual-meeting). This meeting is perhaps the best place to get a broad overview of AI's growing role in radiology, especially from the clinical side. It is also the best place to wander the massive exhibit floor and talk to vendors about their products.

For those more interested in the basic science side of $\mathrm{AI}$ in radiology, the Society for Imaging Informatics in Medicine (SIIM) is the place to go (https://siim.org/ page/upcoming_meetings). SIIM's mission is to advance medical imaging informatics across the enterprise through education, research, and innovation in a multidisciplinary community, placing a strong emphasis on enterprise imaging (strategies, initiatives, and workflows implemented across a healthcare enterprise to consistently and optimally capture, index, manage, store, distribute, view, exchange, and analyze all clinical imaging and multimedia content to enhance the EHR). They also have, in 
association with the American Board of Imaging Informatics, a program to become a certified imaging informatics professional.

There are numerous other conferences to attend, but it is also important to note that the American College of Radiology has a commission on informatics, the American Board of Radiology has incorporated informatics into its board and continuing certification examinations, an increasing number of residency programs have an informatics track as well as informatics fellowships.

\section{Conclusion}

Clearly AI is an inevitable part of radiology's and healthcare's future. What is required to make it successful (i.e., impact patient care and outcomes) is to carefully develop strategies that are proactive in terms of how we control and manage patient data that are used to develop and then implement AI tools (i.e., data are a commodity but how do we make the selling of data palatable to industry, healthcare enterprises, and patients), how and in what circumstances we integrate AI into the clinical workflow, how we educate and train users, how we deal with the still emerging medicolegal issues surrounding AI, and even how we assess the impact of AI on the ultimate users-clinicians. ${ }^{43,44}$

\section{Author Disclosure Statement}

No competing financial interests exist.

\section{Funding Information}

No funding was received for this article.

\section{References}

1. Kohn LT, Corrigan JM, Donaldson MS. To Err is Human: Building a Safer Health System. National Academies Press, Washington, DC: 2000.

2. Institute of Medicine Committee on Quality of Health Care in America. Crossing the Quality Chasm: A New Health System for the 21st Century. National Academies Press, Washington, DC: 2001.

3. Scoglio AAJ, Reilly ED, Gorman JA, et al. Use of social robots in mental health and well-being research: systematic review. J Med Internet Res. 2019; $21: e 13322$.

4. Chamberlain WE. Fluoroscopes and fluoroscopy: Carman Lecture. Radiology. 1942; 38:383-413.

5. Birkelo CC, Chamberlain WE, Phelps PS, et al. Tuberculosis case finding: a comparison of the effectiveness of various roentgenographic and photofluorographic methods. J Am Med Assoc. 1947; 133:359-366.

6. Garland LH. On the scientific evaluation of diagnostic procedures. Radiology. 1949; 52:309-328.

7. Newell RR, Chamberlain WE, Rigler L. Descriptive classification of pulmonary shadows; a revelation of unreliability in the roentgenographic diagnosis of tuberculosis. Am Rev Tuberc. 1954; 69:566-584.

8. Tuddenham WJ, Calvert WP. Visual search patterns in roentgen diagnosis. Radiology. 1961; 76:255-256.

9. Kundel HL, Nodine CF, Carmody D. Visual scanning, pattern recognition and decision-making in pulmonary nodule detection. Invest Radiol. 1978; 13:175-181.

10. Kundel HL, Nodine CF, Krupinski EA. Searching for lung nodules. Visual dwell indicates locations of false-positive and false-negative decisions. Invest Radiol. 1989; 24:472-478.
11. Waite S, Scott J, Gale B, et al. Interpretive error in radiology. AJR Am J Roentgenol. 2017; 208:739-749.

12. Degnan AJ, Ghobadi EH, Hardy $P$, et al. Perceptual and interpretive error in diagnostic radiology-causes and potential solutions. Acad Radiol. 2019; 26:833-845.

13. Busby LP, Courtier JL, Glastonbury CM. Bias in radiology: the how and why of misses and misinterpretations. Radiographics. 2018; 38:236247.

14. Bruno MA, Walker EA, Abujudeh $\mathrm{HH}$. Understanding and confronting our mistakes: the epidemiology of error in radiology and strategies for error reduction. Radiographics. 2015; 35:1668-1676.

15. Krupinski EA, Berbaum KS, Caldwell RT, et al. Do long radiology workdays affect nodule detection in dynamic CT interpretation? J Am Coll Radiol. 2012; 9:191-198.

16. Krupinski EA, Berbaum KS, Caldwell RT, et al. Long radiology workdays reduce detection and accommodation accuracy. J Am Coll Radiol. 2010; 7:698-704.

17. Ruutiainen AT, Durand DJ, Scanlon $M H$, et al. Increased error rates in preliminary reports issued by radiology residents working more than 10 consecutive hours overnight. Acad Radiol. 2013; 20:305-311.

18. Wildman-Tobriner B, Cline B, Swenson C, et al. Evaluating resident on-cal performance: does volume affect discrepancy rate? Curr Probl Diagn Radiol. 2018; 47:364-367.

19. Seidel RL, Krupinski EA. The agony of it all: musculoskeletal discomfort in the reading room. J Am Coll Radiol. 2017; 14:1620-1625.

20. Hanna TN, Lamoureux C, Krupinski EA, et al. Effect of shift, schedule, and volume on interpretive accuracy: a retrospective analysis of 2.9 million radiologic examinations. Radiology. 2018; 287:205-212.

21. Katzen J, Dodelzon K. A review of computer aided detection in mammography. Clin Imaging. 2018; 52:305-309.

22. Oakden-Rayner $L$. The rebirth of CAD: how is modern Al different from the CAD we know? Radiol Artif Intell. 2019; 1:190089.

23. Burt JR, Torosdagli N, Khosravan N, et al. Deep learning beyond cats and dogs: recent advances in diagnosing breast cancer with deep neural networks. Br J Radiol. 2018; 91:20170545.

24. McBee MP, Awan OA, Colucci AT, et al. Deep learning in radiology. Acad Radiol. 2018; 25:1472-1480.

25. Bell LTO, Gandhi S. A comparison of computer-assisted detection (CAD) programs for the identification of colorectal polyps: performance and sensitivity analysis, current limitations and practical tips for radiologists. Clin Radiol. 2018; 73:e11-593.e18.

26. Murphy A, Skalski M. The utilisation of convolutional neural networks in detecting pulmonary nodules: a review. Br J Radiol. 2018; 91:20180028.

27. Nawrocki T, Maldjian PD, Slasky SE, et al. Artificial intelligence and radiology: have rumors of the radiologist's demise been greatly exaggerated? Acad Radiol. 2018; 25:967-972.

28. Bluemke DA. Radiology in 2018: are you working with Al or being replaced by Al? Radiology. 2018; 287:365-366.

29. Gallix B, Chong J. Artificial intelligence in radiology: who's afraid of the big bad wolf? Eur Radiol. 2019; 29:1637-1639.

30. Goldberg JE, Rosenkrantz AB. Artificial intelligence and radiology: a social media perspective. Curr Probl Diagn Radiol. 2019; 48: 308-311.

31. Pintos dos Santos D, Giese D, Brodfehl S, et al. Medical students' attitude towards artificial intelligence: a multicentre survey. Eur Radiol. 2019; 29:1640-1646.

32. Char DS, Shah NH, Magnus D. Implementing machine learning in health care-addressing ethical challenges. N Engl J Med. 2018; 378:981-983.

33. United States Food \& Drug Administration. Proposed regulatory framework for modifications to artificial intelligence/machine learning (Al/ML)-based software as a medical device (SaMD). https:// www.fda.gov/media/122535/download. (Last accessed on August 11 2019).

34. Handelman GS, Kok HK, Chandra RV, et al. Peering into the black box of artificial intelligence: evaluation metrics of machine learning methods. AJR Am J Roentgenol. 2019; 212:38-43.

35. Douzas G, Bacao F. Effective data generation for imbalanced learning using conditional generative adversarial networks. Expert Syst Appl. 2018; 91:464-471. 
36. Vayena E, Blasimme A, Cohen IG. Machine learning in medicine: addressing ethical challenges. PLoS Med. 2019; 15:e1002689.

37. Prior FW, Brunsden B, Hildebolt $C$, et al. Facial recognition from volumerendered magnetic resonance imaging data. IEEE Trans Inf Technol Biomed. 2009; 13:5-9.

38. Rodriguez-Ruiz A, Krupinski E, Mordang JJ, et al. Detection of breast cancer with mammography: effect of an intelligent support system. Radiology. 2019; 290:305-314.

39. Krupinski E, Bronkalla M, Folio L, et al. Advancing the diagnostic cockpit of the future: an opportunity to improve diagnostic accuracy and efficiency. Acad Radiol. 2019; 26:579-581.

40. Pesapane F, Volonte $C$, Codari M, et al. Artificial intelligence as a medical device in radiology: ethical and regulatory issues in Europe and the United States. Insights Imaging. 2018; 9:

$$
\text { 745-753. }
$$

41. Wong SH, Alam Z, Alam A. Artificial intelligence in radiology: how will we be affected? Eur Radiol. 2019; 29:141-143.

42. Gentin A. Israeli handheld ultrasound could revolutionize keeping tabs on breast cancer. https://www.timesofisrael.com/israeli-handheldultrasound-could-revolutionize-keeping-tabs-on-breast-cancer/. (Last accessed on August 11, 2019).

43. Liew $C$. The future of radiology augmented with artificial intelligence: a strategy for success. Eur J Radiol. 2018; 102:152-156.

44. Thrall JH, Li X, Cruz C, et al. Artificial intelligence and machine learning in radiology: opportunities, challenges, pitfalls and criteria for success. J Am Coll Radiol. 2018; 15:504-508. 